\section{Evaluación y manejo inicial de las ideas e intentos de suicidio en atención primaria}

\author{
LILIAN SALVO G. ${ }^{1,2}$, RAMÓN FLORENZANO U. ${ }^{3,4,5}$, \\ ALEJANDRO GÓMEZ CH. ${ }^{6}$
}

\section{Initial assessment and management of the suicidal ideation and suicide attempts in primary care}

Suicide and suicidal behaviors are a significant public health problem, for which several prevention modalities have been implemented. The identification and treatment of suicidal risk is a critical step in prevention, especially in primary health care, a level where most of the population consults. This article focuses fundamentally on the research on suicide risk in primary care by proposing an action guide for the assessment and management initial of suicide ideas and attempts.

(Rev Med Chile 2021; 149: 913-919)

Key words: Primary Health Care; Suicidal Ideation; Suicide, Attempted.
'Servicio de Psiquiatría, Hospital Clínico Herminda Martín, Chillán. Chile. ${ }^{2}$ Facultad de Medicina, Universidad Católica de la Santísima Concepción. Concepción. Chile.

${ }^{3}$ Facultad de Medicina, Campus Oriente, Universidad de Chile.

Santiago. Chile.

${ }^{4}$ Facultad de Medicina, Universidad de los Andes. Santiago. Chile.

${ }^{5}$ Facultad de Psicología, Universidad del Desarrollo. Santiago. Chile.

${ }^{6}$ Departamento de Psiquiatría y Salud Mental, Campus Sur, Facultad de Medicina, Universidad de Chile. Santiago, Chile.

Trabajo no recibió financiamiento. Los autores declaran no tener conflictos de interés.

Recibido el 11 de diciembre de 2020, aceptado el 25 de mayo de 2021

Correspondencia a:

Lilian Salvo G.

Bulnes 473, Chillán.

Isalvog@gmail.com
E 1 suicidio es un fenómeno de etiología multifactorial que constituye un serio problema de salud pública. Para prevenir el suicidio y las conductas suicidas se han implementado y evaluado diversas estrategias universales, selectivas e indicadas ${ }^{1}$. Destacan a nivel de la población: políticas de salud mental, políticas para disminuir el consumo perjudicial de alcohol, acceso a atención en salud, restricción del acceso a métodos letales, recomendaciones a los medios de comunicación y sensibilización sobre salud mental, trastornos por consumo de sustancias y suicidio ${ }^{1-3}$. En grupos de mayor riesgo: líneas telefónicas de ayuda en crisis, capacitación de gatekeepers, programas escolares de prevención del suicidio, acceso oportuno e intervenciones para grupos vulnerables y personas con patología psiquiátrica, y capacitación a médicos en el reconocimiento y tratamiento de la depresión y otras enfermedades psiquiátricas ${ }^{1-3}$. A nivel individual: seguimiento y soporte comunitario, tratamiento de los trastornos psiquiátricos comórbidos y tra- tamientos específicos para la suicidalidad, dentro de los cuales se considera elaborar un plan de seguridad, los tratamientos farmacológicos, las terapias psicológicas e intervenciones psicosociales $^{1-3}$. En el ámbito de la atención sanitaria, los pilares de la prevención del suicidio son la identificación de las personas de alto riesgo, la evaluación psiquiátrica y evaluación del riesgo suicida y las estrategias de manejo oportuno y adecuado $^{3}$.

La consulta en servicios de atención primaria previa a la muerte por suicidio es común. La evidencia muestra que $77 \%$ de las víctimas de suicidio tuvieron contacto con proveedores de atención primaria de salud el año previo y $45 \%$ el mes previo al suicidio ${ }^{4}$, en comparación con servicios de salud mental donde 26 a $32 \%$ estuvo en contacto el año previo y $19 \%$ el mes previo al suicidio $^{4,5}$. Una revisión selectiva de la evidencia sobre las intervenciones en atención primaria para prevenir el suicidio da cuenta de cuatro estrategias principales: capacitación a profesionales, detec- 
ción del riesgo de suicidio y/o de alteración del estado de ánimo, tratamiento de la depresión, y evaluación y manejo del riesgo suicida ${ }^{6}$. También destacan que una sola sesión de intervención de planificación de respuesta a la crisis puede ser eficaz en reducir la ideación y los intentos de suicidio $^{6}$, lo cual es consistente con los resultados de efectividad en reducir el riego de suicidio demostrados por la WHO BIC, una intervención de una sesión inmediata con plan de seguimiento posterior ${ }^{7,8}$. Por tanto y considerando sólo el contexto sanitario, la atención primaria es un entorno importante donde actuar para ayudar a prevenir la suicidalidad.

En relación con lo anterior, el propósito de este artículo es aportar evidencias de la literatura que apoyan el manejo de la prevención y atención de las ideas e intentos suicidas en atención primaria y presentar un protocolo de evaluación y manejo inicial de la suicidalidad en ese nivel, en base a la evidencia, la experiencia clínica y la práctica docente de los autores. Con el objeto de colaborar con la capacitación de los médicos, la detección del riesgo de suicidio y el manejo inicial de las ideas e intentos de suicidio en atención primaria.

\section{Evaluación clínica del riesgo de suicidio}

A la fecha no se cuenta con biomarcadores clínicamente prácticos, pruebas de laboratorio o exámenes de imágenes que ayuden en la valoración del riesgo suicida, por lo tanto la evaluación del paciente suicidal sigue siendo competencia del clínico, quien debe combinar el juicio clínico con el conocimiento empírico, los factores de riesgo y de protección y sopesar éstos para brindar una ayuda integral al paciente individual ${ }^{3,9}$. La evaluación del riesgo de suicidio se realiza como parte de la evaluación médica. En ésta se requiere tener presente la identificación de factores de riesgo y factores protectores ${ }^{9-12}$. En el ambiente sanitario, en todos los niveles de atención, son relevantes los siguientes factores:

\section{Factores de Riesgo}

\section{Antecedente de suicidalidad previa o actual Ideas suicidas de diversa severidad, intentos}

de suicidio previos e historia familiar de conducta suicida $^{1-3,10-15}$.

\section{Presencia de síntomas psicopatológicoso enfermedad psiquiátrica}

Síntomas depresivos, agitación, elevación del ánimo, mayor irritabilidad, alteraciones del sueño, ansiedad, angustia, impulsividad, autoagresiones sin fines suicidas. Trastorno depresivo, trastorno bipolar, trastorno por dependencia al alcohol u otras sustancias, esquizofrenia, trastornos de ansiedad, trastornos de la personalidad, trastornos de la conducta alimentaria, déficit atencional y trastornos conductuales ${ }^{1-3,10,11,15-19}$.

\section{Otros factores que adquieren relevancia} especialmente si se agregan a los anteriores

- Sociodemográficos: adolescentes, adultos mayores, ser hombre sobre 60 años (pero está aumentando la tasa de suicidio en mujeres), vivir solo, pérdida de la pareja, pertenecer a la comunidad LGBTI, inmigración, desempleo, problemas laborales, económicos, legales o ambientales ${ }^{1-3,10,11,20}$.

- Psicológicos y sociales: desesperanza, impulsividad, déficit para resolver problemas, baja autoestima, déficit de apoyo social, disfunción familiar, historia de abuso o trauma especialmente en la infancia, eventos vitales estresantes, pérdida de sentido de pertenencia, percibirse una carga para los demás y dolor psíquico ${ }^{1-3,10,11,21}$.

- Enfermedades médicas: dolor crónico, enfermedades médicas crónicas especialmente de mal pronóstico o que comprometan el funcionamiento ${ }^{1-3,10,11}$.

- Acceso a medios letales ${ }^{1-3,10,11}$.

\section{Factores Protectores}

Sentido de responsabilidad por otros, sentido de pertenencia, soporte social, satisfacción con la vida, habilidades de afrontamiento, habilidades para resolver problemas, habilidades para testear la realidad, religiosidad, percepción de conexión, vínculo terapéutico y otros de importancia para el paciente ${ }^{3,11,22}$. 


\section{Evaluación y manejo de las ideas suicidas en atención primaria}

El riesgo suicida es claramente mayor en personas que presentan ideas suicidas ${ }^{13}$. Pero, rara vez las personas consultan espontáneamente por estas ideas. Por lo tanto, en el ámbito de la atención primaria todos los profesionales de salud deberían tratar de pesquisar la presencia de ideación suicida, mientras realizan controles de salud, atenciones de morbilidad y/o atenciones de urgencia ${ }^{23-25}$.

Dada la mayor prevalencia de ideación suicida en determinados grupos ${ }^{1-3,6,10-21,24,25}$ es recomendable focalizarse en esos grupos de riesgo. Entonces:

\section{Indagar por ideas suicidas}

Se recomienda indagar en mayor profundidad las ideas suicidas en los siguientes grupos:

- Personas que consultan espontáneamente por síntomas psicopatológicos o si éstos se hicieron presentes cuando el clínico consultó por ellos o se hicieron evidentes en el examen: ánimo deprimido, desesperanza, mayor irritabilidad, elevación del ánimo, autoagresiones, angustia, ansiedad, alteraciones del sueño, impulsividad, agresividad, desórdenes conductuales, trastornos de la conducta alimentaria.

- Personas con antecedentes de intentos de suicidio.

- Personas con diagnóstico de enfermedades psiquiátricas.

- Personas con consumo indebido de alcohol u otras sustancias.

- Personas que estén viviendo alguna situación o evento estresante psicosocial y/o ambiental y/o con antecedente de trauma en la infancia.

- Personas con enfermedades médicas o quirúrgicas crónicas, dolorosas, malignas, de mal pronóstico o que comprometen el funcionamiento.

- Adolescentes, especialmente si son impulsivos, con problemas conductuales o más aún si presentan algunas de las condiciones antes citadas
- Adultos mayores, especialmente con desmedro socioeconómico y enfermedades.

- Personas con antecedente de conducta suicida en la familia.

\section{Modo de consultar por ideas suicidas y procedimientos a efectuar}

En ambiente de entrevista cálido, respetuoso y confidencial, consultar progresivamente y proceder de acuerdo al nivel de suicidalidad encontra$\mathrm{do}^{1,3,10,24,25}$, como se presenta en la Figura 1.

\section{Evaluación y manejo del intento de suicidio en atención primaria}

Frente a una persona que ha realizado un intento de suicidio, la premura del actuar médico será guiada por la presencia o no de urgencia vital, procediendo ${ }^{1,3,10,24,25}$ a:

1. Evaluar y actuar para resolver las consecuencias médico-quirúrgicas.

2. Evaluar y actuar para resolver el estado de urgencia psiquiátrica.

3. Realizar evaluación clínica psiquiátrica.

4. Decidir plan disposicional.

5. Iniciar tratamiento y seguimiento.

Todo paciente que realiza un intento de suicidio requiere ser evaluado por psiquiatra. La evaluación, manejo y derivación a unidad de hospitalización o a programa ambulatorio se presenta en la Figura 2 y Figura 3.

\section{Recomendaciones finales}

La evaluación y manejo de la conducta suicida debe ser incorporada en la práctica rutinaria no sólo en psiquiatría sino que también en medicina general. Requiere considerar al paciente, la familia $\mathrm{y} / \mathrm{u}$ otras personas significativas, actuando con un enfoque multidimensional y un abordaje multidisciplinario que incluya evaluación sistemática del riesgo suicida, planes de seguridad, tratamientos biológicos, psicológicos, psicosociales y de seguimiento, con estrecha coordinación entre atención primaria y de especialidad. 


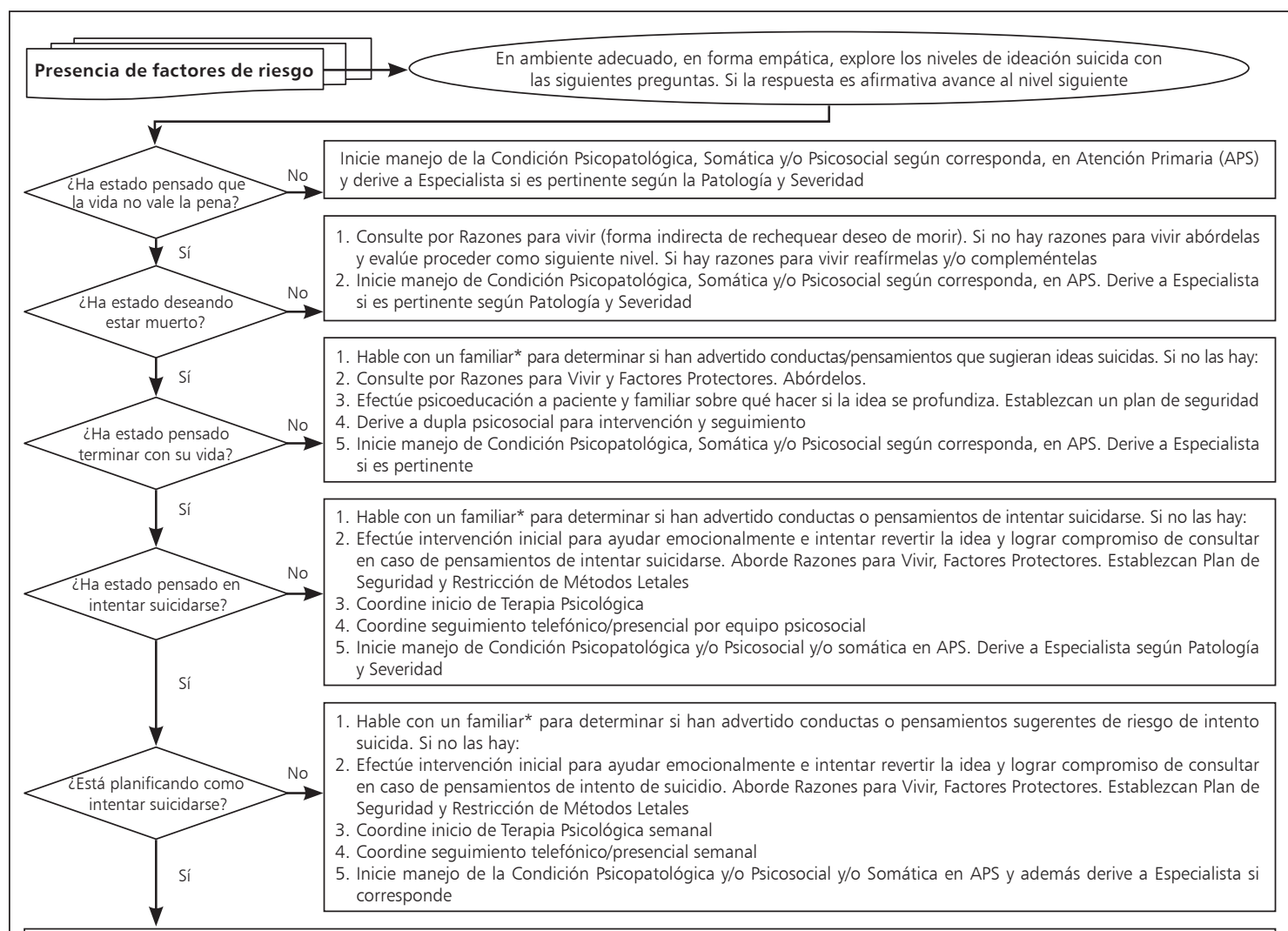

Realice intervención para intentar revertir la ideación suicida. Si lo logra proceda como en nivel anterior. Si no lo logra: derive con supervisión y medidas anti intento de suicidio a evaluación psiquiátrica en el mismo día a Policlínico de Choque o Unidad de Emergencia.

Nota: Frente a dudas proceda como nivel de mayor severidad.

*Si no cuenta con familiar, contactar red de apoyo.

Figura 1. Flujograma de manejo de la ideación suicida en atención primaria.

Intervención inicial: intervención cálida, atenta y de confianza, con comunicación franca en que se escucha empáticamente, se permite expresión de las emociones y sentimientos y se ofrece ayuda. Se exploran los motivos de la ideación o intento de suicidio y se revisan las posibilidades de abordarlos. Se intenta revertir la idea suicida y lograr compromiso de tratamiento y de consulta en caso de riesgo de intento de suicidio. Se abordan las razones para vivir y los factores protectores $7,8,25$. Plan de seguridad: intervención que incluye una lista priorizada de estrategias de afrontamiento y fuentes de apoyo y consulta a las cuales acudir en caso de riesgo suicida. Individualizado según cada paciente y construido en conjunto entre médico, paciente y familiar (o tutor o responsable de la red de apoyo) 3,26-29. En conjunto proceder a: 1) enseñar a reconocer las señales de advertencia (emociones, estados de ánimo, pensamientos, imágenes, comportamientos) cuando comienza a pensar en el suicidio o siente impulso o se siente extremadamente angustiado; 2 ) revisar las estrategias de afrontamiento interno a las que el paciente recurrirá para no actuar según sus emociones, pensamientos o impulsos; 3) acordar y registrar entornos sociales seguros y enumerar varias personas (por si primera opción no esté disponible) que pueden distraerlo de la crisis por si ocurriera que con el propio afrontamiento no puede resolverla o reducir el riesgo de intentar suicidarse; 4) revisar a que familiares o amigos podría recurrir para que le ayude a resolver la crisis si con contactos sociales no fue posible, enumerar a quien solicitará ayuda en lista priorizada (varios por si primera opción no esté disponible) e identificar posibles obstáculos y la forma como superarlos; 5) educar y registrar contacto de profesionales de salud y unidades de urgencia (nombres, ubicación, lugar, vía de acceso, teléfono) donde recurrir si la crisis no se resuelve o reduce el riesgo de intento de suicidio y 6) indagar sobre potenciales medios letales y acordar colaborativamente el modo de restringir el acceso. Restricción métodos letales en ámbito de atención de salud: indague con el paciente y familia tenencia o presencia de métodos suicidas en el hogar o trabajo: armas de fuego, pesticidas, herbicidas, fármacos almacenados u otros ${ }^{1-3,25}$. Acordar el retiro de dichos métodos. Considerar la presencia de situaciones estructurales de riesgo suicida (por ejemplo, ventanas o balcones en altura, lugares desde los cuales podría intentar el ahorcamiento). Si es necesaria la prescripción de fármacos, entregar éstos en cantidad adecuada a familiar u otro responsable quien administrará el tratamiento. Si los medicamentos prescritos tienen un nivel elevado de letalidad, advertirlo al familiar u otro responsable, para que tome los resguardos apropiados. Seguimiento: intervención estructurada realizada por profesionales de salud capacitados que consiste en llamadas telefónicas y/o visitas (según requerimiento del estado psicopatológico, social o somático del paciente) en que se evalúa el riesgo suicida, se interviene según el estado psicopatológico del paciente, se revisa el plan de seguridad, se apoya el compromiso con el tratamiento y se coordina la red según necesidad7,8,25,28. Post intervención inicial se realiza semanalmente, a futuro se podrá ir distanciando según la evolución del paciente. Derivación a especialista: cuando corresponde derivar al paciente según la patología psiquiátrica que presente y su severidad, de acuerdo con las recomendaciones de las guías clínicas ministeriales de nuestro país (Depresión ${ }^{30}$, Trastorno Bipolar ${ }^{31}$, Esquizofrenia ${ }^{32}$, Consumo perjudicial y dependencia de alcohol y otras drogas 33 y los protocolos de derivación de patologías psiquiátricas de cada Servicio de Salud; y cuando el paciente presente ideas suicidas con riesgo de que efectúe intento de suicidio. 


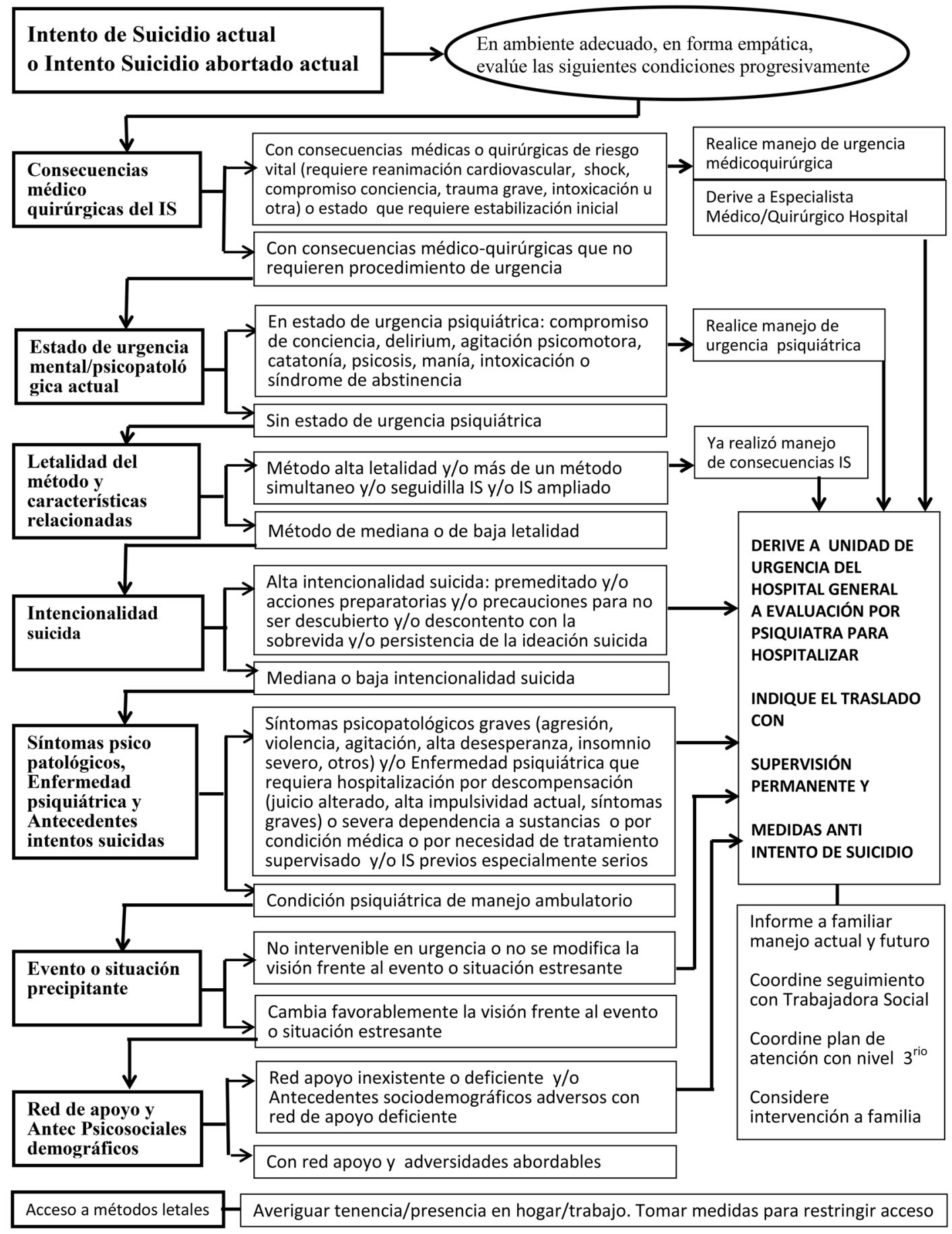

Figura 2. Protocolo para decidir en APS derivación a hospitalización por intento de suicidio. 
Intento de suicidio actual

o Intento de suicidio abortado actual

Con $\downarrow$

Consecuencias médico-quirúrgicas leves de resolución ambulatoria en atención primaria o que no requieren tratamiento

Sin estado de urgencia psiquiátrica

Método suicida de mediana o de baja letalidad

Mediana o baja intencionalidad suicida que luego de intervención está arrepentido(a) de su intento o persiste con deseos de morir pero no intentaría suicidarse y está dispuesto a iniciar y cooperar con el tratamiento y buscar ayuda y/o consultar si la idea suicida se intensifica o vuelve a pensar en posibilidad de intentar suicidarse

Condición psiquiátrica de manejo ambulatorio y sin intentos previos médicamente serios

Cambia favorablemente la visión frente al evento o situación que motivó el intento suicida

Su situación de vida es estable y cuenta con red de apoyo

Se cuenta con médico para indicar tratamiento, equipo psicosocial para intervenciones y seguimiento y con psiquiatra en nivel secundario donde derivar
1. Efectuar intervención para ayudar emocionalmente, abordar la crisis y revertir ideas suicidas

\section{Abordar razones para vivir y factores protectores}

3. Acordar plan de seguridad y
compromiso de consultar SOS

4. Acordar restricción de acceso a métodos letales

5. Iniciar tratamiento de la condición psicopatológica o trastorno psiquiátrico comórbido. Si requiere fármacos los administrará el familiar

\section{Abordar situaciones precipitantes}

7. Coordinar intervención psicológica

8. Coordinar seguimiento semanal

9. Realizar derivación a psiquiatra ambulatorio en nivel secundario

10. Coordinar plan de atención con el nivel secundario

11. Considerar intervención a la familia

Figura 3. Protocolo para decidir en APS derivación a manejo ambulatorio por intento de suicidio.

\section{Referencias}

1. WHO. Preventing suicide A global imperative. Disponible en https://www.who.int/mental_health/suicide-prevention/world_report_2014/es/. [Consultado el 2 de octubre de 2020].

2. Fazel S, Runeson B. Suicide. N Engl J Med. 2020; 382: 266-74.

3. Ryan E, Oquendo M. Suicide risk assessment and prevention: Challenges and opportunities. Focus 2020; 18: 88-99.

4. Luoma J, Martin C, Pearson J. Contact with mental health and primary care providers before suicide: A review of the evidence. Am J Psychiatry 2002: 159: 909-16.

5. Walby F, Myhre MO, Kildahl AT. Contact with mental health services prior to suicide: A systematic review and meta-analysis. Psychiatr Serv. 2018; 69: 751-9.

6. Dueweke AR, Bridges AJ. Suicide interventions in pri- mary care: A selective review of the evidence. Fam Syst Health. 2018; 36 (3): 289-302.

7. Fleischmann A, Bertolote J, Wasserman D, De Leo D, Bolhari J, Botega N, et al. Effectiveness of brief intervention and contact for suicide attempters: a randomized controlled trial in five countries. Bull World Health Organ. 2008; 86 (9): 703-9.

8. Riblet N, Shiner B, Young Y, Watts B. Strategies to prevent death by suicide: meta-analysis of randomized controlled trials. Br J Psychiatry 2017; 210 (6): 396-402.

9. Gómez A. Manejo y tratamiento integral. En: Gómez A, Silva H, Amon R, Editores, El suicidio. Teoría y clínica. Santiago, Chile: Editorial Mediterráneo Ltda 2018; 18698.

10. American Psychiatric Association. Practice guideline for the assessment and treatment of patients with suicidal behaviors. Disponible en https://psychiatryonline.org/ pb/assets/raw/sitewide/practice_guidelines/guidelines/ suicide.pdf. [Consultado el 9 de octubre de 2020]. 
11. Suicide Prevention Resource Center. A guide for primary care providers and medical practice managers. Disponible en https://www.sprc.org/sites/default/files/ Section $\% 202 \% 20$ Education $\% 20$ Clinicians $\% 20$ and $\% 20$ Office\%20Staff.pdf. [Consultado el 9 de octubre de 2020].

12. Weber A, Michail M, Thompson A, Fiedorowicz J. Psychiatric emergencies: Assessing and Managing suicidal ideation. Med Clin North Am. 2017; 101 (3): 553-71.

13. Hubers A, Moaddine S, Peersmann, Stijnen T, Duijn E, Vander Mast R, et al. Suicidal ideation and subsequent completed suicide in both psychiatric and non-psychiatric populations: a meta-analysis. Epidemiol Psychiatr Sci. 2018; 27 (2): 186-98.

14. Hill NTM, Robinson J, Pirkis J, Andriessen K, Krysinska $\mathrm{K}$, Payne A, et al. Association of suicidal behavior with exposure to suicide and suicide attempt: A systematic review and multilevel meta-analysis. Plos Med. 2020; 17 (3): e1003074.

15. Ribeiro JD, Franklin JC, Fox KR, Bentley KH, Kleiman EM, Chang BP, et al. Self-injurious thughts and behaviors as risk factors for future suicide ideation, attempts, and death: a meta-analysis of longitudinal studies. Psychol Med. 2016; 46 (2): 225-36.

16. San Too L, Spittal M, Bugeja L, Reifels L, Butterworth P, Pirkis J. The association between mental disorders and suicide: A systematic review and meta-analysis of record linkage studies. J Affect Disord. 2019; 259: 302-13.

17. Salvo L, Ramírez J, Castro A. Factores de riesgo para intento de suicidio en presonas con trastorno depresivo en atención secundaria. Rev Med Chile 2019; 147: 181-9.

18. Ribeiro J, Huang X, Fox K, Franklin J. Depression and hopelessness as risk factors suicide ideation, attempts and death: meta-analysis of longitudinal studies. Br J Psychiatry 2018; 212 (5): 279-86.

19. Bruzzone C, Reynal J, Florenzano R, Juejati M, Tolosa P, Tapia A. Estudio piloto de la aplicación del protocolo SUPRE MISS de la OMS para la evaluación de pacientes suicidas hospitalizados. Rev Chil Neuro-Psiquiat. 2006; 60: 84.

20. Huang X, Ribeiro JD, Musacchio KM, Franklin JC. Demographics as predictors of suicidal thoghts and behaviors: A meta-analysis. Plos ONE 2017; 12 (7): e0180793.

21. Batty GD, Kivimaki M, Bell S, Gale C, Shipley M, Whitley E, et al. Psychosocial characteristics as potential predictors of suicide in adults an overview with new from prospective cohort studies. Transl Psychiatry 2018; 8,22.

22. Florenzano R. La relevancia de la espiritualidad en la prevención del suicidio. En: Gómez A, Silva H, Amon R, Editores, El suicidio. Teoría y clínica. Santiago, Chile: Editorial Mediterraneo Ltda. 2018; 221-8.
23. Florenzano R, Acuña J, Fullerton C, Castro C. Comparative study of the frequency and features of emotional disturbances in patients in primary care clinics. Rev Med Chile 1998; 126 (4): 397-405.

24. Gómez A. Evaluación del riesgo de suicidio: enfoque actualizado. Rev Med Clin Condes 2012; 23 (5): 607 15.

25. Ministerio de Salud. Programa nacional de prevención del suicidio: orientaciones para su implementación. Santiago, Chile: Minsal 2013. Disponible en: https://www. minsal.cl/sites/default/files/Programa_Nacional_Prevencion.pdf. [Consultado el 9 de octubre de 2020].

26. Brown G, Stanley B. Safety Planning Guide: A Quick Guide for Clinicians. Disponible https://www.sprc.org/ resources-programs/safety-planning-guide-quick-guide-clinicians. [Consultado el 9 de octubre de 2020].

27. Stanley B, Brown GK. Safety planning intervention: a brief intervention to mitigate suicide risk. Cogn Behav Pract. 2012; 19: 256-64.

28. Stanley B, Brown GK, Brenner L, Galfalvy H, Currier G, KnoxK, et al. Comparison of the safety planning intervention with follow-up vs care of suicidal patients treated in the emergency department. JAMA Psychiatry 2018; 75 (9). 894-900.

29. Labouliere CD, Stanley B, Lake AM, Gould M S. Safety planning on crisis lines: feasibility, acceptability, and perceived helpfulness of a brief intervention to mitigate future suicide risk. Suicide Life Threat Behav. 2020; 50: 29-41.

30. Ministerio de Salud. Guía Clínica Depresión en personas de 15 años y más. Santiago, Chile: Minsal 2013. Disponible en: https://www.minsal.cl/portal/url/item/ 7222754637c08646e04001011f014e64.pdf. [Consultado el 7 de noviembre de 2020].

31. Ministerio de Salud. Guía de Práctica Clínica de Trastorno Bipolar en personas de 15 años y más. Santiago, Chile: Minsal 2018. Disponible en: https://www.minsal.cl/ portal/url/item/db835d0231c5115fe0400101640126b7. pdf. [Consultado el 7 de noviembre de 2020].

32. Ministerio de Salud. Guía Clínica para el tratamiento de personas desde el primer episodio de Esquizofrenia. Santiago, Chile: Minsal 2017. Disponible en: https://diprece.minsal.cl/wrdprss_minsal/wp-content/ uploads/2017/07/GPC_EQZ.pdf. [Consultado el 7 de noviembre de 2020].

33. Ministerio de Salud. Guía de Práctica Clínica Consumo Perjudicial y Dependencia de Alcohol y otras Drogas en personas menores de 20 años. Santiago, Chile: Minsal 2018. Disponible en: https://www.minsal.cl/portal/url/ item/7222c0667853b8f8e04001011f016146.pdf. [Consultado el 7 de noviembre de 2020]. 